\title{
Circular RNA expression profiles in the development of acute chronic liver failure in hepatitis $B$ virus- infected patients
}

\author{
Jiangshan Lian \\ zhejiang university \\ Feiyan Lin \\ zhejiang university \\ Yinfeng Lu \\ zhejiang university \\ Shaorui Hao \\ zhejiang university \\ Jiong Yu \\ zhejiang university \\ Huan Cai \\ zhejiang university \\ Xiaoli Zhang \\ zhejiang university \\ Yuqing Gu \\ zhejiang university \\ Yida Yang \\ zhejiang university \\ lufeng hu ( $\nabla$ hulufeng79@sina.com ) \\ wenzhou medical university
}

\section{Research article}

Keywords: High-throughput RNA sequencing technology, circRNA, hepatitis B virus, chronic hepatitis B, acute chronic liver failure

Posted Date: September 18th, 2020

DOI: https://doi.org/10.21203/rs.3.rs-38521/v2

License: (1) This work is licensed under a Creative Commons Attribution 4.0 International License. Read Full License 


\section{Abstract}

Hundreds of millions of people worldwide suffer from chronic hepatitis $B(C H B)$, which is thus among the most serious public health problems. CHB can lead to serious acute chronic liver failure (ACLF). Since ACLF has a very high mortality rate, prediction of ACLF risk is a critical issue in clinical practice. To investigate the development of ACLF from CHB, circular RNA (circRNA), a novel type of endogenous non-coding RNA, was examined in healthy control $(\mathrm{HC})$ subjects and $\mathrm{CHB}$ and ACLF patients using high-throughput RNA sequencing technology. Differentially expressed circRNAs were selected and analyzed by Gene Ontology (GO) and Kyoto Encyclopedia of Genes and Genomes (KEGG) biological pathway analyses, circRNA-miRNA coexpression networks, and statistical analyses. The results showed that a total of 21,101 circRNAs were identified in $\mathrm{HC}, \mathrm{CHB}$ and $\mathrm{ACLF}$ subjects. Compared with the HCs, 4 up-regulated and 14 down-regulated circRNAs were identified in ACLF subjects, which exhibited a consistent trend of change in the three groups. $\mathrm{GO}$ analysis revealed that regulation of lymphocyte activation and macrophage tolerance induction were the most important biological processes in ACLF progression. KEGG pathway analysis revealed that primary immunodeficiency and NOD-like receptor signaling pathways were the most enriched terms. The circRNAmiRNA co-expression network and statistical analyses showed that circRNA_07734, circRNA_08533, and circRNA_16083 were the most important circRNAs in the process of ACLF. In conclusions, immune dysfunction and immunodeficiency may be critical in the development of ACLF, which results in aberrant expression of circRNAs, especially circRNA_07734, circRNA_08533, and circRNA_16083.

\section{Background}

Chronic hepatitis $B(\mathrm{CHB})$ is one of the most serious public health problems worldwide. More than 2 billion people have been infected with hepatitis $B$ virus (HBV), 360 million of whom are chronically infected [1]. Although CHB is initially asymptomatic, it can lead to serious acute chronic liver failure (ACLF, HBV-ACLF) after several years, which has a poor prognosis and a high mortality rate $(>70 \%)$ [2]. ACLF is serious in CHB patients, who constitute approximately $70 \%$ of all ACLF cases in most Eastern countries [2, 3]. In China, ACLF patients account for approximately $80 \%$ of all ACLF cases [4]. Since ACLF has a very high mortality rate, prediction of ACLF risk is a major issue in clinical practice.

At present, the model for end-stage liver disease (MELD) is recognized as a reliable method to measure the mortality risk of patients with liver failure. Shen et al. assessed six widely used short- and long-term prognostic models for patients with ACLF. They concluded that the integrated MELD model may be optimal [5]. Liu et al. suggested that incorporating the gamma-glutamyl transpeptidase to platelet ratio into the MELD model may allow more accurate prediction of survival in ACLF patients [6]. Ma et al established a scoring system offering superior predictive performance, in terms of both specificity and sensitivity, for ACLF patients compared with the MELD [7]. Wei et al. reported that serum Golgi protein 73 (GP73) may be useful in the diagnosis of ACLF in populations with chronic HBV infections [8]. Zhu et al. evaluated the prognostic utility of serum Hcy levels, which may serve as a biomarker for 3-month mortality rate in ACLF patients [9]. Although many studies predicting the risk of ACLF have been performed, no method had been proven effective and the pathogenesis of ACLF remains unclear. 
Circular RNA (circRNA) is a novel endogenous non-coding RNA characterized as a widespread, abundant, stable, conserved and tissue-specific molecule in mammalian cells [10]. Different from linear RNA, circRNA is not degraded by RNase R; it is composed of a class of RNA developing covalently closed loop structures without 5'-3' polarities [10, 11]. CircRNAs are important competing endogenous RNAs. They act as sponges for microRNA (miRNA) and regulate the expression of miRNA-targeted transcripts. Moreover, circRNAs have the potential to serve as biomarkers for the diagnosis and prognosis of several cancers $[12,13]$. For example, circRNAs associated the occurrence and development of hepatocellular carcinoma (HCC) [14], such as circRNA_100338 and circRNA_0005075, serve as biomarkers for HCC, and as targets for HCC therapeutics associated with HBV [15-17].

The numbers of circular RNAs are different between CHB and ACLF, and increase with symptom severity [18]. So far, there has been no report on circRNAs in CHB and ACLF, and whether aberrant expression of circRNAs plays a role in ACLF is still unknown. Our study aimed to demine the numbers of circRNAs in CHB and ACLF, and to identify the important circRNAs in ACLF development.

\section{Methods}

\subsection{Clinical samples and ethics statement}

The current study was approved by the Medical Ethics Committee of the First Affiliated Hospital, School of Medicine, Zhejiang University (Registry number 201743), China. Three CHB and three ACLF patients admitted to the Liver Center were included. Three healthy controls (HCs) were recruited from the Physical Examination Center. All clinical examinations and data collection procedures were conducted in accordance with the Declaration of Helsinki.

\subsection{Total RNA isolation}

Blood samples $(5 \mathrm{~mL})$ were collected into heparinized tubes and diluted 2:1 with phosphate-buffered saline. Mononuclear cells (MNCs) were obtained with equal amounts $\left(1.077 \mathrm{~g} / \mathrm{cm}^{3}\right.$ ) of Ficoll-Paque (Ficoll-Pague ${ }^{\mathrm{Tm}}$ PLUS; GE Healthcare, Chicago, IL, USA) and density gradient centrifugation.

Total RNA was extracted using QIAamp ${ }^{\circledR}$ RNA Blood Mini (Qiagen, No: 52304). The concentration and purity of RNA were detected on NanoDrop ND-1000 spectrophotometer (Thermo Fisher Scientific, Inc.). The ratio of the readings at $260 \mathrm{~nm}$ and $280 \mathrm{~nm}$ (A260/A280) was measured. For accurate values, we calibrated the spectrophotometer with the same solution and measured absorbance in $10 \mathrm{mM} \mathrm{Tris} \cdot \mathrm{Cl},+\mathrm{pH}$ 7.5. And the quality of extracted RNA was detected by using Agilent 4200 TapeStation system (G2991AA) according to the Agilent RNA ScreenTape Assay Quick Guide for 4200 TapeStation System.RNA integrity was evaluated using the Agilent 4200 Bioanalyzer (Agilent Technologies, Santa Clara, CA, USA). Samples with an RNA integrity number (RIN) $\geq 7$ were subsequently subjected to analysis.

\subsection{High-throughput RNA sequencing of circRNAs and mRNA}

The concentration and purity of total RNA extracted from MNCs were measured on a NanoDrop ND-1000 spectrophotometer (Thermo Fisher Scientific, Inc.) at 260 and $280 \mathrm{~nm}$ wavelengths. Before the synthesis of 
first- and second-strand cDNA, the ribo-Zero (ribosomal RNA) was depleted and RNA-fragmented. CDNA was purified followed by processing of adenylate 3 ' ends, ligation of adapters and enrichment of DNA fragments. Finally, $1 \mu \mathrm{L}$ was loaded onto a 2100 Bioanalyzer (Agilent Technologies) for validation. High-throughput RNA sequencing was performed according to the method of Memczak et al [19]. The experimental process is shown in Supplemental Figure 1.

\subsection{Identification of differentially expressed circRNAs and mRNA}

Raw data were extracted using Agilent Feature Extraction software and predicted by circBase. circRNAs and mRNA were quantitatively analyzed by Shanghai OE Biotech Ltd., Co. (Shanghai, China). The circRNAs and mRNA of the HC, CHB and ACLF subjects were verified with CIRI software (Shanghai OE Biotech) and the identified sequence were selected for further analysis. The expression levels of circRNAs among the three

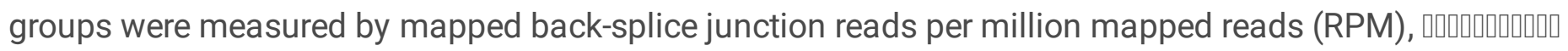

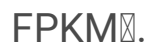

Using the DESeq package (https://ngs.csr.uky.edu/DESeq), which uses a model based on the negative binomial distribution method, was applied to detect differentially expressed circRNAs and mRNA. The location of the chromosome where the circRNA sequence overlapped was annotated. The criteria were chosen according to the fold change and p-value of the circRNA and mRNA in the same sample between two groups.

\subsection{Gene Ontology (GO) and Kyoto Encyclopedia of Genes and Genomes (KEGG) pathway analyses}

DAVID (Database for Annotation, Visualization and Integrated Discovery; http://www.david.abcc.ncifcrf.gov/) was used to analyze the potential functions of the linear transcripts. Gene functions were classified into three subgroups: biological process (BP), cellular component (CC) and molecular function (MF). The most enriched GO terms among the CHB and ACLF groups (ranked by enrichment score) were selected, and the enriched GO terms between $\mathrm{HC}$ and ACLF were ranked by the number of differentially expressed linear transcripts. The different biological pathways of linear transcripts were analyzed by the KEGG pathway.

\section{6 miRNA target prediction}

circRNA can be used as a target molecule of miRNA and regulated by miRNA. Analysis of the circRNA-miRNA interaction could help elucidate the function and mechanism of circRNAs in contact with miRNAs. Therefore, the homemade miRNA target prediction software, Arraystar (Rockville, MD, USA), which is based on miRanda (www. microrna.org/microrna/home.do) and Target Scan (www.targetscan.org/vert_71), was used to predict the circRNA-miRNA interactions, to further investigate the functional roles of the differentially expressed circRNAs related to CHB and ACLF. The target interaction network diagram of the circRNA-miRNA/gene interaction network was built by Cytoscape (available on: http://www.cytoscape.org/).

\subsection{Statistical analyses}

The clinical parameters and expression levels of mRNA in the HC, CHB and ACLF groups are presented as means \pm standard deviation (SD). Statistically significant differences among the three groups were analyzed by analysis of variance (ANOVA) and LSD post-test. Independent sample t-test was used to analyze the expression of selected circRNA (RPM) between HC and ACLF groups. Spearman's correlation was performed 
to evaluate the relation between circRNA with mRNA and liver function indexes. All statistical analyses were conducted with SPSS software (ver. 16.0; IBM Corp., Armonk, NY, USA) and P<0.05 was considered statistically significant.

\section{Results}

\subsection{Clinical information}

Albumin (ALB), globulin (GLO), alanine transaminase (ALT), aspartate aminotransferase (AST), cholinesterase (CHE), alkaline phosphatase (ALP), total bilirubin (TBIL), direct bilirubin (DBIL), indirect bilirubin (IBIL), glutamyl transferase (GGT), creatinine (Cr), alpha fetoprotein (AFP), prothrombin time (PT), international normalized ratio (INR), white blood cell (WBC), hemoglobin (Hb), and platelet count (PLT), were measured and analyzed by ANOVA. ALB, ALT, CHE, ALP, TBIL, DBIL, IBIL, PT, and INR differed significantly $(\mathrm{P}<0.05)$ among the $\mathrm{HC}, \mathrm{CHB}$ and ACLF groups (Table 1).

Table 1. Clinical parameters in the HC, CHB and ACLF groups and ANOVA statistics

\begin{tabular}{llcccc}
\hline Parameter & Unit & CHB & ACLF & HC & ANOVA \\
\hline Age & years & $39.33 \pm 4.73$ & $40.33 \pm 7.02$ & $33.67 \pm 3.21$ & 0.313 \\
ALB & $\mathrm{g} / \mathrm{L}$ & $40.90 \pm 0.44$ & $35.20 \pm 2.16$ & $51.11 \pm 1.00$ & $<0.001$ \\
$\mathrm{GLO}$ & $\mathrm{g} / \mathrm{L}$ & $24.60 \pm 3.40$ & $25.93 \pm 6.49$ & $27.53 \pm 1.12$ & 0.716 \\
$\mathrm{ALT}$ & $\mu \mathrm{mol} / \mathrm{L}$ & $112.33 \pm 149.47$ & $853.00 \pm 138.62$ & $14.00 \pm 1.00$ & $<0.001$ \\
$\mathrm{AST}$ & $\mu \mathrm{mol} / \mathrm{L}$ & $36.67 \pm 19.76$ & $304.67 \pm 271.88$ & $10.00 \pm 1.73$ & 0.112 \\
$\mathrm{CHE}$ & $\mathrm{U} / \mathrm{L}$ & $7782.33 \pm 1050.24$ & $5092.00 \pm 361.96$ & $8136.00 \pm 485.45$ & 0.003 \\
$\mathrm{ALP}$ & $\mathrm{U} / \mathrm{L}$ & $65.00 \pm 11.79$ & $163.00 \pm 52.60$ & $52.33 \pm 3.21$ & 0.009 \\
$\mathrm{TBIL}$ & $\mu \mathrm{mol} / \mathrm{L}$ & $14.00 \pm 12.49$ & $425.67 \pm 172.69$ & $14.00 \pm 2.65$ & 0.003 \\
$\mathrm{DBIL}$ & $\mu \mathrm{mol} / \mathrm{L}$ & $6.33 \pm 5.86$ & $251.67 \pm 93.18$ & $2.00 \pm 1.00$ & 0.002 \\
$\mathrm{IBIL}$ & $\mu \mathrm{mol} / \mathrm{L}$ & $7.67 \pm 6.66$ & $174.00 \pm 94.87$ & $12.00 \pm 2.65$ & 0.016 \\
$\mathrm{GGT}$ & $\mu \mathrm{mol} / \mathrm{L}$ & $48.67 \pm 54.50$ & $92.67 \pm 38.89$ & $15.67 \pm 1.53$ & 0.125 \\
$\mathrm{Cr}$ & $\mu \mathrm{mol} / \mathrm{L}$ & $75.67 \pm 17.21$ & $59.67 \pm 15.04$ & $71.67 \pm 4.04$ & 0.376 \\
$\mathrm{AFP}$ & $\mu \mathrm{g} / \mathrm{L}$ & $6.33 \pm 4.29$ & $83.47 \pm 64.43$ & $3.27 \pm 0.49$ & 0.065 \\
$\mathrm{PT}$ & $\mathrm{s}$ & $11.77 \pm 0.87$ & $38.50 \pm 5.30$ & $11.14 \pm 0.89$ & $<0.001$ \\
$\mathrm{INR}$ & - & $1.03 \pm 0.08$ & $3.68 \pm 0.49$ & $1.01 \pm 0.05$ & $<0.001$ \\
$\mathrm{WBC}$ & $10^{9} / \mathrm{L}$ & $5.73 \pm 1.37$ & $6.20 \pm 1.87$ & $5.80 \pm 0.46$ & 0.904 \\
$\mathrm{Hb}$ & $\mathrm{g} / \mathrm{L}$ & $151.33 \pm 24.01$ & $148.33 \pm 5.13$ & $160.00 \pm 3.61$ & 0.610 \\
$\mathrm{PLT}$ & $10^{9} / \mathrm{L}$ & $164.67 \pm 21.01$ & $103.00 \pm 39.04$ & $207.67 \pm 16.29$ & 0.009 \\
\hline
\end{tabular}

\subsection{Overview of circRNA profiles}

High-throughput sequencing revealed a total of 21,101 circRNAs in the HC, CHB and ACLF subjects. Among the 21,101 circRNAs, 13,024 were detected in HCs, 13,172 in CHB patients, 12,166 in ACLF patients, and 6,445 in all three groups (Figure. 1B). There are no outliers of circRNA expression in the $\mathrm{HC}$, $\mathrm{CHB}$, or ACLF group due to instrument or operational anomalies. The distribution of circRNAs on the genome is shown in Supplemental Figure 2.

Approximately $86.61 \%$ of circRNAs had a predicted spliced length of less than $2,000 \mathrm{nt} ; 48.44 \%$ had a length less than 500 nt and $26.53 \%$ had a length between 500 nt and 1,000 nt (Figure. 1A). 


\subsection{Identification of differentially expressed circRNAs and mRNA}

According to the circRNA criteria (fold change $>2$ and difference of $\mathrm{P}<0.05$ between two groups), 550 differentially expressed circRNAs in the CHB and ACLF groups were identified compared with the HC group. Among them, 176 differentially expressed circRNAs were selected in the CHB group (89 up-regulated and 87 down-regulated) and 374 differentially expressed circRNAs were selected in the ACLF group (181 up-regulated and 193 down-regulated). The differentially expressed circRNAs between the ACLF and HC groups are shown in a volcano plot (Figure. 2A). The unsupervised hierarchical cluster analysis results are shown in Figure 2B.

The differentially expressed candidate circRNAs among the three groups showing progressive up- or downregulation were further analyzed. There were 4 up-regulated and 14 down-regulated circRNAs (Table 2, Figure 3). mRNAs were analyzed by the same method. The results showed there were 7 up-regulated and 9 downregulated mRNA. The expression information and statistic differences of circRNA and mRNA were shown in the Supplemental Table 1,2.

Table 2. Differentially expressed circRNAs (ACLF vs. HC)

\begin{tabular}{|c|c|c|c|c|c|c|c|c|c|}
\hline \multirow[t]{2}{*}{ CircRNA } & \multicolumn{2}{|c|}{ CHB vs. HC } & \multicolumn{2}{|c|}{ ACLF vs. HC } & \multirow[t]{2}{*}{ Regulation } & \multirow[t]{2}{*}{ Chromosome } & \multirow[t]{2}{*}{ Length } & \multirow[t]{2}{*}{ Strand } & \multirow[t]{2}{*}{ Type } \\
\hline & $\mathrm{FC} 2$ & P_val & $\mathrm{FC} 2$ & P_val & & & & & \\
\hline circRNA_00389 & 1.6887 & 0.1679 & 3.5835 & 0.0006 & down & chr1 & 363 & + & $\begin{array}{l}\text { sense- } \\
\text { overlapping } \\
\text { sense- }\end{array}$ \\
\hline circRNA_02808 & 1.6185 & 0.4137 & 3.6685 & 0.0080 & down & $\operatorname{chr} 10$ & 361 & - & overlapping \\
\hline circRNA_03646 & 1.3686 & 0.4386 & 5.8769 & 0.0003 & down & chr11 & 6085 & - & intergenic \\
\hline circRNA_04205 & 1.5300 & 0.2323 & 3.2813 & 0.0112 & down & chr12 & 1368 & - & overlapping \\
\hline circRNA_05781 & 1.7071 & 0.1450 & 3.2003 & 0.0131 & down & $\operatorname{chr} 14$ & 3199 & - & intergenic \\
\hline circRNA_06400 & 1.5643 & 0.1009 & 3.3205 & 0.0000 & down & $\operatorname{chr} 14$ & 369 & - & exonic \\
\hline circRNA_07485 & 1.9656 & 0.1531 & 3.2457 & 0.0107 & down & chr16 & 845 & + & exonic \\
\hline circRNA_07734 & 1.7126 & 0.1422 & 2.8129 & 0.0077 & down & chr16 & 313 & + & overlapping \\
\hline circRNA_08533 & 2.7321 & 0.0232 & 13.2602 & 0.0000 & down & $\operatorname{chr} 17$ & 939 & + & $\begin{array}{l}\text { Sense- } \\
\text { overlapping }\end{array}$ \\
\hline circRNA_09331 & 1.4930 & 0.2115 & 7.4732 & 0.0004 & down & chr18 & 658 & + & exonic \\
\hline circRNA_17523 & 1.5966 & 0.1871 & 3.7990 & 0.0074 & down & chr6 & 640 & - & overlapping \\
\hline circRNA_17526 & 2.4262 & 0.0631 & 6.1841 & 0.0091 & down & chr6 & 439 & - & $\begin{array}{l}\text { sense- } \\
\text { overlapping }\end{array}$ \\
\hline circRNA_19214 & 1.8125 & 0.1170 & 7.6139 & 0.0001 & down & chr8 & 258 & - & $\begin{array}{l}\text { sense- } \\
\text { overlapping }\end{array}$ \\
\hline circRNA_04487 & 0.5663 & 0.2698 & 4.4060 & 0.0010 & up & chr12 & 455 & + & $\begin{array}{l}\text { Sense- } \\
\text { overlapping }\end{array}$ \\
\hline circRNA_15699 & 0.6673 & 0.3637 & 3.6601 & 0.0351 & up & chr5 & 1923 & + & exonic \\
\hline circRNA_16083 & 0.7123 & 0.5781 & 5.4313 & 0.0000 & up & chr5 & 5262 & + & exonic \\
\hline circRNA_16987 & 0.8639 & 0.8767 & 7.9830 & 0.0000 & up & chr6 & 527 & - & overlapping \\
\hline
\end{tabular}

Note: FC, fold-change, absolute ratio (non-log scale) of normalized intensities between two groups (threshold, 2.0). P-value calculated using a paired t-test (threshold, 0.05). circRNA, circular RNA; hsa, Homo sapiens; +, positive strand; -, negative strand.

\subsection{GO and KEGG analyses}

The functions of the target genes of differentially expressed circRNAs in the ACLF group were further analyzed by GO enrichment and KEGG pathway analysis. Significant terms in the GO and KEGG pathway analyses $(P<0.05)$ were selected and ranked by $p$-value. 
The top-10 down-regulated GO terms, classified by BP, CC, MF, are shown in Supplemental Figure 3A. The most enriched BP, CC and MF terms were regulation of lymphocyte activation, integrin alphaL-beta2 complex and RNA polymerase I core binding, respectively. Among the up-regulated GO terms, the most enriched BP, CC and MF terms were positive regulation of macrophage tolerance induction, Golgi lumen, and extracellular matrix structural constituent, respectively (Supplemental Figure. 3B).

KEGG pathway analysis (Table 3, Supplemental Figure 4 ) revealed that the most enriched term in downregulated circRNAs was primary immunodeficiency, while in up-regulated circRNAs, NOD-like receptor signaling pathway was the most enriched term. As shown in Table 3, KEGG pathway hsa05340 was the most important. The KEGG map is shown in Supplemental Figure 5.

Table 3. KEGG analysis of ACLF risk status in up- and down-regulated circRNAs ranked by p-value and enrichment score

\begin{tabular}{lcccl}
\hline CircRNA & Path-id & P(10-3) & Core & Term \\
\hline \multicolumn{2}{l}{ Down-regulated circRNAs } & & & \\
circRNA_00389 & hsa05340 & 0.0424 & 91.525 & Primary immunodeficiency \\
circRNA_00389 & hsa05166 & 0.0177 & 11.5368 & HTLV-I infection \\
circRNA_07734 & hsa05150 & 0.1249 & 53.8382 & Staphylococcus aureus infection \\
circRNA_00389 & hsa04650 & 0.1724 & 14.0808 & Natural killer cell-mediated cytotoxicity \\
circRNA_07734 & hsa05144 & 0.2829 & 35.8922 & Malaria \\
circRNA_07734 & hsa05323 & 0.3921 & 30.5083 & Rheumatoid arthritis \\
circRNA_08533 & hsa05219 & 0.3921 & 30.5083 & Bladder cancer \\
circRNA_07734 & hsa05416 & 0.9218 & 19.8967 & Viral myocarditis \\
circRNA_08533 & hsa05230 & 1.5902 & 15.1281 & Central carbon metabolism in cancer \\
circRNA_07734 & hsa04514 & 1.7781 & 14.3008 & Cell adhesion molecules (CAMs) \\
Up-regulated circRNAs & & & \\
circRNA_15699 & hsa04621 & 0.4187 & 29.5242 & NOD-like receptor signaling pathway \\
circRNA_16083 & hsa04514 & 1.7781 & 14.3008 & Cell adhesion molecules (CAMs) \\
circRNA_16987 & hsa04915 & 3.1911 & 10.6424 & Estrogen signaling pathway \\
circRNA_04487 & hsa04722 & 8.5407 & 6.4454 & Neurotrophin signaling pathway \\
\hline
\end{tabular}

\section{5 circRNA-miRNA co-expression network}

A network map of the most significant relationships between circRNAs and miRNAs is shown in Figure 4. Among the differentially expressed circRNAs selected from the ACLF group, circRNA_03646, circRNA_06400, circRNA_09331, circRNA_17523, circRNA_05781, circRNA_07734, circRNA_08533, circRNA_16562, circRNA_17526, circRNA_15699, circRNA_16083 underpinned the circRNA-miRNA co-expression network, as determined by Arraystar. Among them, thee expression of circRNA_03646, circRNA_06400, circRNA_07734, circRNA_08533, circRNA_16083 had statistical significance between ACLF and HC group. Moreover, circRNA_07734, circRNA_08533, and circRNA_16083 were also had statistical significance in KEGG analysis. circRNA_07734 only contacted two miRNA in the network, which less than circRNA_08533 and circRNA_16083.

\subsection{Correlation analysis}

Spearman's correlation analysis indicated that the RPM values ofcircRNA_07734, circRNA_08533, and circRNA_16083 were related to ALT, AST, TBIL, and IBIL (Table 4). circRNA_07734 andcircRNA_08533 showed 
strong negative correlation with ALT, AST, TBIL, and IBIL, which indicated if ALT, AST, TBIL, and IBIL increased higher, the expression of circRNA_07734 and circRNA_08533 would be lower. circRNA_16083 is different with circRNA_07734 and circRNA_08533, which had positive correlation with ALT, AST, TBIL, and IBIL. In clinical practice, ALT, AST, TBIL, and IBIL are used to evaluate the liver function. Commonly, the higher the ALT, AST, TBIL, and IBIL level were, the more severe the liver injury will be. Therefore, circRNA_07734, circRNA_08533, and circRNA_16083 may be useful as the sensitive indicators of liver injury.

Table 4. Spearman's correlations analysis of circRNA_07734, circRNA_08533, and circRNA_16083 with differentially expressed mRNA and alanine transaminase (ALT), aspartate aminotransferase (AST), total bilirubin (TBIL), and indirect bilirubin (IBIL) $(\mathrm{n}=9)$

\begin{tabular}{|c|c|c|c|c|c|c|}
\hline \multirow{2}{*}{$\begin{array}{l}\text { mRNA and } \\
\text { liver index }\end{array}$} & \multicolumn{2}{|c|}{ circRNA_07734 } & \multicolumn{2}{|c|}{ circRNA_08533 } & \multicolumn{2}{|c|}{ circRNA_16083 } \\
\hline & Coefficient & $\mathrm{P}$ & Coefficient & $\mathrm{P}$ & Coefficient & $\mathrm{P}$ \\
\hline NM_000361.2 & -0.417 & 0.265 & $-0.700 *$ & 0.036 & 0.65 & 0.058 \\
\hline NM_001005862.2 & $.733^{*}$ & 0.025 & $0.800 * *$ & 0.01 & $-0.733^{*}$ & 0.025 \\
\hline NM_001199139.1 & $-.667 *$ & 0.05 & $-0.867 * *$ & 0.002 & 0.65 & 0.058 \\
\hline NM_004633.3 & $-.683 *$ & 0.042 & $-0.883 * *$ & 0.002 & $0.667 *$ & 0.05 \\
\hline NM_005204.3 & $-.750 *$ & 0.02 & $-0.883 * *$ & 0.002 & $0.750 *$ & 0.02 \\
\hline NM_006720.3 & $.783^{*}$ & 0.013 & $0.867 * *$ & 0.002 & $-0.900 * *$ & 0.001 \\
\hline NM_014790.4 & $.700 *$ & 0.036 & $0.950 * *$ & $<0.001$ & $-0.733^{*}$ & 0.025 \\
\hline NM_080913.3 & $-.833 * *$ & 0.005 & $-0.983 * *$ & $<0.001$ & $0.833 * *$ & 0.005 \\
\hline XM_005257807.2 & $.733 *$ & 0.025 & $0.917 * *$ & 0.001 & $-0.733 *$ & 0.025 \\
\hline XM_011511061.1 & $-.817 * *$ & 0.007 & $-0.967 * *$ & $<0.001$ & $0.850 * *$ & 0.004 \\
\hline XM_011520156.1 & -0.267 & 0.488 & -0.317 & 0.406 & 0.5 & 0.17 \\
\hline XM_011520271.1 & 0.567 & 0.112 & $0.833^{* *}$ & 0.005 & -0.617 & 0.077 \\
\hline XM_011524254.1 & 0.633 & 0.067 & 0.583 & 0.099 & $-0.717^{*}$ & 0.03 \\
\hline XM_011524699.1 & $.717 *$ & 0.03 & $0.800 * *$ & 0.01 & $-0.750 *$ & 0.02 \\
\hline XM_011531698.1 & $-.800 * *$ & 0.01 & $-0.867 * *$ & 0.002 & $0.883^{* *}$ & 0.002 \\
\hline XM_011537524.1 & 0.517 & 0.154 & 0.6 & 0.088 & -0.533 & 0.139 \\
\hline ALT & $-.733^{*}$ & 0.025 & $-.733^{*}$ & 0.025 & $.733 *$ & 0.025 \\
\hline AST & -0.469 & 0.203 & -0.603 & 0.086 & $.669 *$ & 0.049 \\
\hline TBIL & -0.583 & 0.099 & $-.883^{* *}$ & 0.002 & $.767 *$ & 0.016 \\
\hline IBIL & -0.65 & 0.058 & $-.883 * *$ & 0.002 & $.850 * *$ & 0.004 \\
\hline
\end{tabular}

\section{Discussion}

circRNAs have received increasing attention due to their highly stable and specific spatiotemporal expression patterns. circRNAs are a transcriptional product in various tissue and cell types in humans, mice, drosophila, and nematodes, among other species. circRNAs can be classified into three types: exonic (circRNA arising from the exons of the linear transcript); intergenic (circRNA located outside of a known gene locus); and sense-overlapping (circRNA whose gene locus overlaps with linear RNA but is transcribed from the opposite strand). The differentially expressed hosting genes of the circRNAs and the disordered splicing machinery will lead to circRNAs differentially expressed. So far, numerous circRNAs have been reported in different animals. The majority of circRNAs showing specific expression patterns are related to tissue development and disease [19]. Therefore, circRNA has potential as a biomarker for some diseases [11]. Thus far, many studies have reported abnormal expression levels of circRNAs in a number of cancers, such as gastric [20, 21], colorectal $[22,23]$, breast $[24,25]$, and lung cancers $[26,27]$. However, few studies have focused on the development of ACLF. 
In this study, we detected several circRNAs in HC, CHB and ACLF subjects. The circRNAs were initially identified by reference to a database or website. circBase, (http://circbase.org/) which includes merged genomic data on circRNAs from humans, mice, nematode worms, pike, and coelacanth, provides evidence of their expression[28]. Therefore, we identified the circRNAs detected herein using circBase. A flow diagram of circRNA prediction is shown in Supplemental Figure 6.

CHB is mainly caused by aberrant cellular immunity, due for example to natural killer (NK) cells, cytotoxic T cells and antibody-dependent lymphocytes. When infected with hepatitis $B$, three types of lymphocytes target antigens of the hepatocyte membrane: HBsAg, HBcAg, and other immunoregulatory cells. Auxiliary and inhibitory $T$ cells take part in the immune response, which can lead to immune disorder hypofunction. Many researchers have reported dysfunction and inhibition of T cells in CHB. For example, Raziorrouh et al. [29] reported that tetramer+CD4+ T cell numbers were reduced during $\mathrm{CHB}$. Zhang et al. reported that HBV-specific T cell responses to recombinant HBV core protein were reduced in CHB patients and positively correlated with HBV viral load in co-infected, chronic HBV patients [30]. Therefore, the progression of ACLF from CHB may be related to the BP of immunodeficiency or dysfunction, which can in turn be caused by HTLV-I infection, Staphylococcus aureus infection or other diseases. The process of immunity dysfunction may be related to the NOD-like receptor signaling pathway, the NF-kappa B signaling pathway, and/or the interleukin-1-mediated signaling pathway.

Since circRNAs contain multiple miRNA-binding sites, the function of individual circRNAs can be predicted by their miRNA target gene, and annotated according to the function of their miRNA target gene. The circRNAmiRNA co-expression network identified two up-regulated circRNAs and nine down-regulated circRNAs in this study. After combining the results from the GO and KEGG analyses, circRNA_00389, circRNA_07734, circRNA_08533, circRNA_15699, and circRNA_16083 were identified as the most important circRNAs during the progression of ACLF. Among them, circRNA_00389 had the highest enrichment score in the KEGG analysis, consistent with the $\mathrm{GO}$ analysis. The top term in $\mathrm{BP}$ analysis was regulation of lymphocyte activation, which corresponded to down-regulated circRNA_00389. However, the expression of circRNA_00389 had no statistical significance between ACLF and HC group. Therefore, combined with the results of spearman's correlation analysis, circRNA_07734, circRNA_08533, and circRNA_16083, which are related to immune dysfunction and correlate with ALT, AST, TBIL, and IBIL, are important circRNAs in ACLF.

However, our study had limitation, there are only 3 samples in each group. Therefore, in the further work, we should perform qRT-PCR to determine the expression levels of these circRNAs and carry out research on mechanisms.

\section{Conclusion}

In this work, 21,101 circRNAs were detected in CHB, HC and ACLF groups. In total, 4 up-regulated and 14 down-regulated circRNAs were identified in the ACLF group compared with the HC group, which showed a consistent trend of change in the three groups. GO and KEGG biological pathway analyses, and circRNAmiRNA co-expression network and statistical analyses, revealed that circRNA_07734, circRNA_08533, and circRNA_16083 are the most important circRNAs in ACLF. Their RPM values correlated with ALT, AST, TBIL, and IBIL; thus, immune dysfunction or immunodeficiency may be critical for ACLF development. 


\section{Abbreviations}

Chronic hepatitis B (CHB), hepatitis B virus (HBV), acute chronic liver failure (ACLF, HBV-ACLF), healthy control $(\mathrm{HC})$, Gene Ontology (GO), Kyoto Encyclopedia of Genes and Genomes (KEGG), model for end-stage liver disease (MELD), Golgi protein 73 (GP73), Circular RNA (circRNA), microRNA (miRNA), hepatocellular carcinoma (HCC), Mononuclear cells (MNCs), RNA integrity number (RIN), biological process (BP), cellular component (CC) and molecular function (MF). standard deviation (SD) receiver operating characteristic (ROC) , albumin (ALB), globulin (GLO), alanine transaminase (ALT), aspartate aminotransferase (AST), cholinesterase (CHE), alkaline phosphatase (ALP), total bilirubin (TBIL), direct bilirubin (DBIL), indirect bilirubin (IBIL), glutamyl transferase (GGT), creatinine (Cr), alpha fetoprotein (AFP), prothrombin time (PT), international normalized ratio (INR), white blood cell (WBC), hemoglobin ( $\mathrm{Hb})$, platelet count (PLT).

\section{Declarations}

\subsection{Competing Interests}

The authors declare no conflict of interest.

\subsection{Funding}

This work was supported by fund of Natural Science Foundation of Zhejiang province (LY16H300005), the Scientific Research Projects of Wenzhou Science and Technology Bureau (Y20180106).

\subsection{Availability of data and materials}

The datasets generated and/or analyzed during the current study are not publicly available but are available from the corresponding author on reasonable request.

\subsection{Ethics approval and consent to participate}

The study was approved by the Medical Ethics Committee of the First Affiliated Hospital, School of Medicine, Zhejiang University (Registry number 201743) and all participants were volunteering to participate in this study and all signed the informed consent. And the protocol was performed in accordance with the approved guidelines.

\subsection{Consent for publication}

Not applicable.

\subsection{Authors' contributions}

YY and LH were involved in the planning and the coordination of the study. $\mathrm{JL}, \mathrm{YL}, \mathrm{SH}, \mathrm{JY}, \mathrm{HC}$, $\mathrm{XZ}$, and $\mathrm{YG}$ carried out the experiment and conducted most of data collection. JL and JY supervised the experiment. LH conducted the statistical analysis and wrote the paper. YY conducted the submission. All authors read and approved the final manuscript. 
Not applicable.

\section{References}

1. Taye S, Abdulkerim A, Hussen M: Prevalence of hepatitis B and C virus infections among patients with chronic hepatitis at Bereka Medical Center, Southeast Ethiopia: a retrospective study. $B M C$ research notes 2014, 7:272.

2. Zhou N, Wang K, Fang S, Zhao X, Huang T, Chen H, Yan F, Tang Y, Zhou H, Zhu J: Discovery of a Potential Plasma Protein Biomarker Panel for Acute-on-Chronic Liver Failure Induced by Hepatitis B Virus. Frontiers in physiology 2017, 8:1009.

3. Lei Z, Mo Z, Zhu J, Pang X, Zheng X, Wu Z, Wang K, Li X, Xie D, Gao Z: Soluble ST2 plasma concentrations predict mortality in HBV-related acute-on-chronic liver failure. Mediators of inflammation 2015, 2015:535938.

4. Wan Z, Wu Y, Yi J, You S, Liu H, Sun Z, Zhu B, Zang H, Li C, Liu F et al: Combining serum cystatin C with total bilirubin improves short-term mortality prediction in patients with HBV-related acute-on-chronic liver failure. PloS one 2015, 10(1):e0116968.

5. Shen Y, Liu YM, Wang B, Zhu YG, Wang YY, Wang XL, Ji JL, Shao JG, Qin Y, Qin G: External validation and comparison of six prognostic models in a prospective cohort of HBV-ACLF in China. Annals of hepatology 2016, 15(2):236-245.

6. Liu L, Lan Q, Lin L, Lu J, Ye C, Tao Q, Cui M, Zheng S, Zhang X, Xue Y: Gamma-glutamyl transpeptidaseto-platelet ratio predicts the prognosis in HBV-associated acute-on-chronic liver failure. Clinica chimica acta; international journal of clinical chemistry 2018, 476:92-97.

7. Ma K, Guo W, Han M, Chen G, Chen T, Wu Z, Yang D, Huang J, Huang Y, Zhao X et al: Entecavir treatment prevents disease progression in hepatitis $B$ virus-related acute-on-chronic liver failure: establishment of a novel logistical regression model. Hepatology international 2012, 6(4):735-743.

8. Wei H, Zhang J, Li H, Ren H, Hao X, Huang Y: GP73, a new marker for diagnosing HBV-ACLF in population with chronic HBV infections. Diagnostic microbiology and infectious disease 2014, 79(1):19-24.

9. Cui JW, Xu Y, Wang Y, Gao YX, Guo S, Wang M, Lu X, Yu S, Ma Y, Yuan D et al: Efficacy of initial haemopurification strategy for acute paraquat poisoning in adults: study protocol for a randomised controlled trial (HeSAPP). BMJ Open 2018, 8(6):e021964.

10. Peng L, Yuan XQ, Li GC: The emerging landscape of circular RNA ciRS-7 in cancer (Review). Oncology reports 2015, 33(6):2669-2674.

11. Li L, Guo J, Chen Y, Chang C, Xu C: Comprehensive CircRNA expression profile and selection of key CircRNAs during priming phase of rat liver regeneration. BMC genomics 2017, 18(1):80.

12. Wang $M$, Yang $Y, X u J$, Bai W, Ren $X$, Wu H: CircRNAs as biomarkers of cancer. a meta-analysis. $B M C$ cancer 2018, 18(1):303.

13. Ding HX, Lv Z, Yuan $Y, X u$ Q: The expression of circRNAs as a promising biomarker in the diagnosis and prognosis of human cancers: a systematic review and meta-analysis. Oncotarget 2018, 9(14):11824- 
11836.

14. Cui S, Qian Z, Chen Y, Li L, Li P, Ding H: Screening of up- and downregulation of circRNAs in HBV-related hepatocellular carcinoma by microarray. Oncology letters 2018, 15(1):423-432.

15. Hung CH, Hu TH, Lu SN, Kuo FY, Chen CH, Wang JH, Huang CM, Lee CM, Lin CY, Yen YH et al: Circulating microRNAs as biomarkers for diagnosis of early hepatocellular carcinoma associated with hepatitis B virus. International journal of cancer 2016, 138(3):714-720.

16. Shang X, Li G, Liu H, Li T, Liu J, Zhao Q, Wang C: Comprehensive Circular RNA Profiling Reveals That hsa_circ_0005075, a New Circular RNA Biomarker, Is Involved in Hepatocellular Crcinoma Development. Medicine 2016, 95(22):e3811.

17. Huang XY, Huang ZL, Xu YH, Zheng Q, Chen Z, Song W, Zhou J, Tang ZY, Huang XY: Comprehensive circular RNA profiling reveals the regulatory role of the circRNA-100338/miR-141-3p pathway in hepatitis B-related hepatocellular carcinoma. Scientific reports 2017, 7(1):5428.

18. Ji F, Yang B, Peng X, Ding H, You H, Tien P: Circulating microRNAs in hepatitis B virus-infected patients. Journal of viral hepatitis 2011, 18(7):e242-251.

19. Memczak S, Jens M, Elefsinioti A, Torti F, Krueger J, Rybak A, Maier L, Mackowiak SD, Gregersen LH, Munschauer $\mathrm{M}$ et al: Circular RNAs are a large class of animal RNAs with regulatory potency. Nature 2013, 495(7441):333-338.

20. Chen J, Li Y, Zheng Q, Bao C, He J, Chen B, Lyu D, Zheng B, Xu Y, Long Z et al: Circular RNA profile identifies circPVT1 as a proliferative factor and prognostic marker in gastric cancer. Cancer letters 2017, 388:208-219.

21. Li P, Chen H, Chen S, Mo X, Li T, Xiao B, Yu R, Guo J: Circular RNA 0000096 affects cell growth and migration in gastric cancer. British journal of cancer 2017, 116(5):626-633.

22. Weng W, Wei Q, Toden S, Yoshida K, Nagasaka T, Fujiwara T, Cai S, Qin H, Ma Y, Goel A: Circular RNA ciRS-7-A Promising Prognostic Biomarker and a Potential Therapeutic Target in Colorectal Cancer. Clinical cancer research : an official journal of the American Association for Cancer Research 2017, 23(14):3918-3928.

23. Zhu M, Xu Y, Chen Y, Yan F: Circular BANP, an upregulated circular RNA that modulates cell proliferation in colorectal cancer. Biomedicine \& pharmacotherapy = Biomedecine \& pharmacotherapie 2017, 88:138144.

24. Liang HF, Zhang XZ, Liu BG, Jia GT, Li WL: Circular RNA circ-ABCB10 promotes breast cancer proliferation and progression through sponging miR-1271. American journal of cancer research 2017, 7(7):1566-1576.

25. Lu WY: Roles of the circular RNA circ-Foxo3 in breast cancer progression. Cell cycle 2017, 16(7):589-590.

26. Tian F, Yu CT, Ye WD, Wang Q: Cinnamaldehyde induces cell apoptosis mediated by a novel circular RNA hsa_circ_0043256 in non-small cell lung cancer. Biochemical and biophysical research communications 2017, 493(3):1260-1266.

27. Zeng Y, Xu Y, Shu R, Sun L, Tian Y, Shi C, Zheng Z, Wang K, Luo H: Altered expression profiles of circular RNA in colorectal cancer tissues from patients with lung metastasis. International journal of molecular medicine 2017, 40(6):1818-1828. 
28. Glazar P, Papavasileiou P, Rajewsky N: circBase: a database for circular RNAs. Rna 2014, 20(11):16661670.

29. Raziorrouh B, Heeg M, Kurktschiev P, Schraut W, Zachoval R, Wendtner C, Wachtler M, Spannagl M, Denk G, Ulsenheimer A et al: Inhibitory phenotype of HBV-specific CD4+ T-cells is characterized by high PD-1 expression but absent coregulation of multiple inhibitory molecules. PloS one 2014, 9(8):e105703.

30. Zhang $X$, Xing $H$, Feng $X$, Zhang $H$, Wang $Y$, Yan H: Hepatitis B virus (HBV)-specific T-cell responses to recombinant HBV core protein in patients with normal liver function and co-infected with chronic HBV and human immunodeficiency virus 1 (HIV-1). Virology journal 2013, 10:232.

\section{Figures}
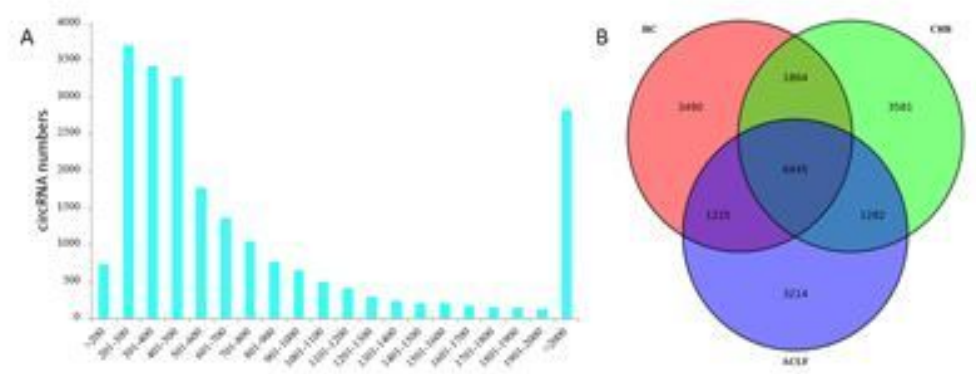

circhesa kength

\section{Figure 1}

Expression patterns of circRNAs in HC, CHB and ACLF subjects. a) Length distribution of circRNAs. b) Venn analysis of circRNAs detected at each time point.
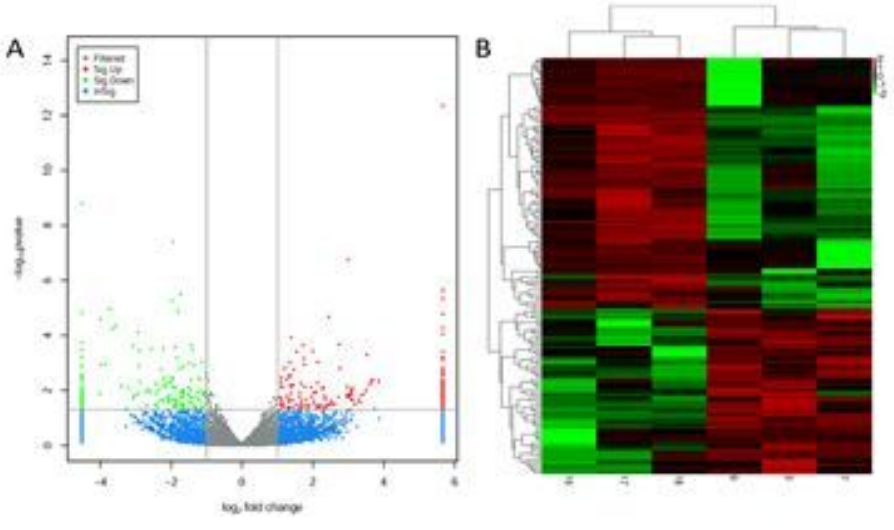

Figure 2

Volcano plot of differentially expressed circRNAs (A) and hierarchical cluster of circRNA expression profiles (B) between ACLF and HC groups 


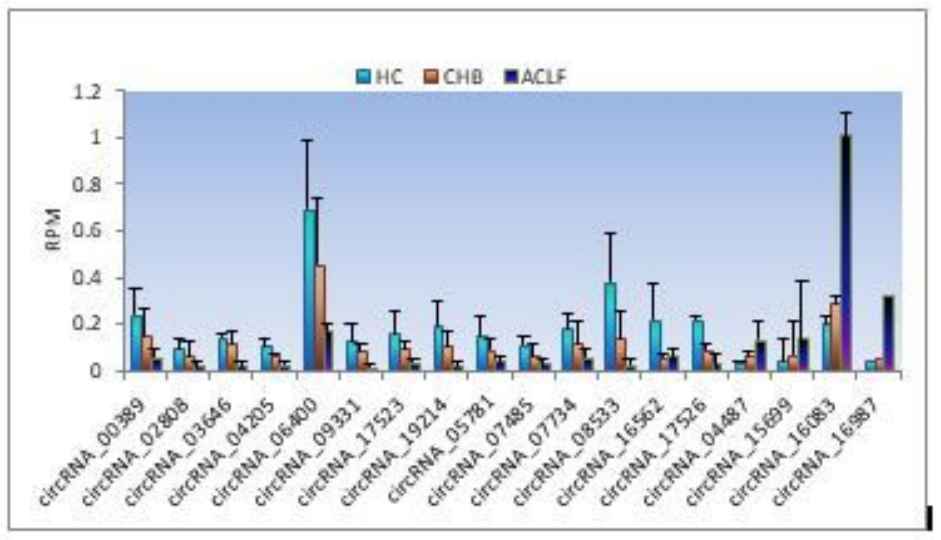

\section{Figure 3}

Differentially expressed (4 up- and 13 down-regulated) circRNAs in the $\mathrm{HC}, \mathrm{CHB}$ and ACLF groups.

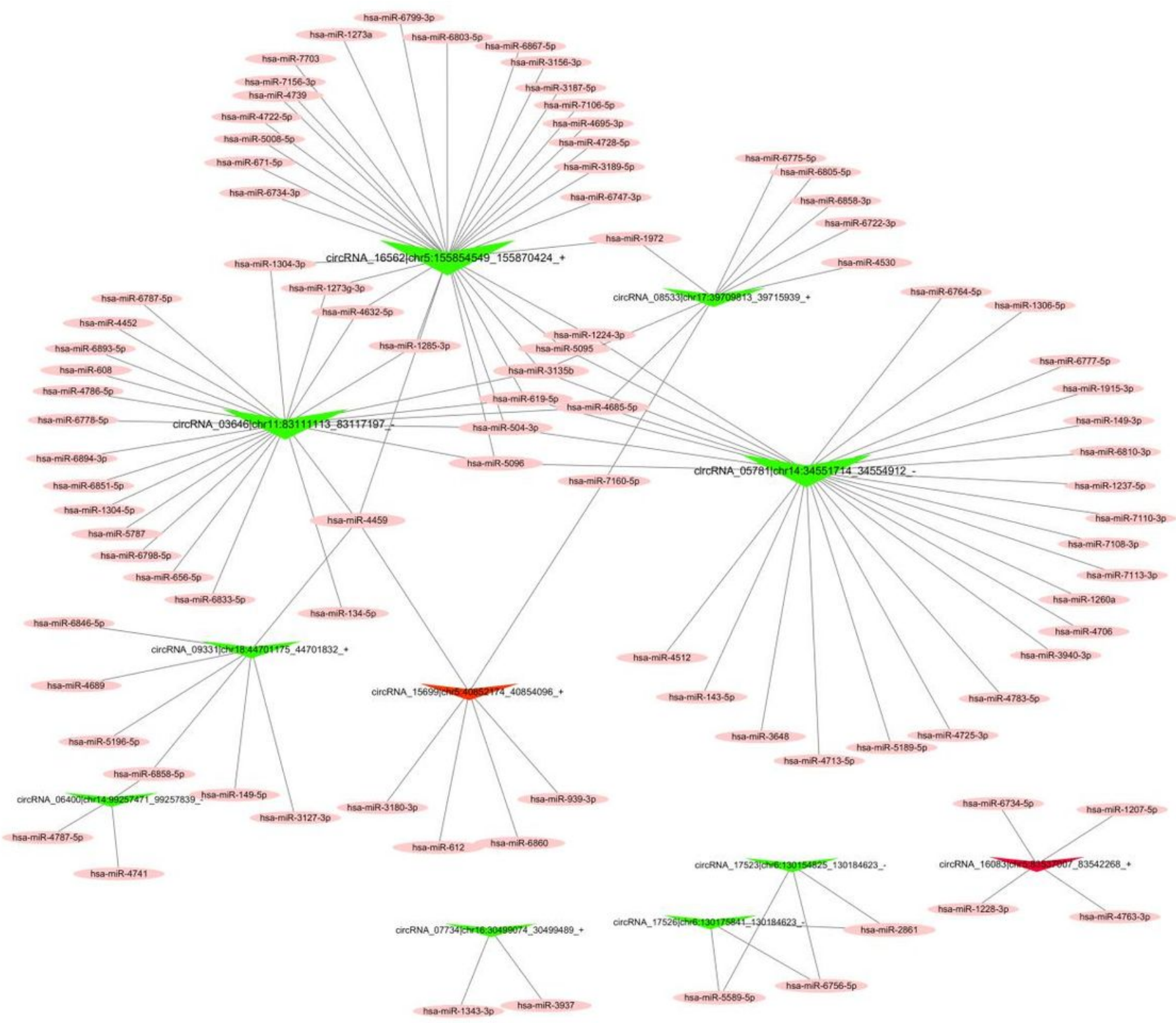


circRNA-miRNA co-expression network based on the selected 18 circRNAs (ACLF vs. HC). Triangle nodes represent circRNAs (red: up, green: down) and circle nodes represent microRNAs (miRNAs).

\section{Supplementary Files}

This is a list of supplementary files associated with this preprint. Click to download.

- SupplementalFile.pdf 